\title{
Anodic Bonded Graphene
}

\author{
Adrian Balan ${ }^{1}$, Rakesh Kumar ${ }^{1}$, Mohamed Boukhicha ${ }^{1}$, Olivier \\ Beyssac $^{1}$, Jean-Claude Bouillard ${ }^{1}$, Dario Taverna ${ }^{1}$, William \\ Sacks $^{1}$, Massimiliano Marangolo ${ }^{2}$, Emanuelle Lacaze ${ }^{2}$, Walter \\ Escoffier $^{3}$, Jean-Marie Poumirol ${ }^{3}$ and Abhay Shukla ${ }^{1}$ \\ ${ }^{1}$ Université Pierre et Marie Curie-Paris 6, CNRS-UMR7590, Institut de Minéralogie \\ et de Physique des Milieux Condensés, 140 rue de Lourmel, Paris, F-75015 France \\ 2 Université Pierre et Marie Curie-Paris 6, CNRS-UMR7588, Institut des \\ Nanosciences de Paris, 140 rue de Lourmel, Paris, F-75015 France \\ 3 Laboratoire National des Champs Magnétiques Intenses, INSA UPS CNRS, UPR \\ 3228, Université de Toulouse, 143 avenue de Rangueil, 31400 Toulouse, France \\ E-mail: abhay.shukla@upmc.fr
}

\begin{abstract}
We show how to prepare graphene samples on a glass substrate with the anodic bonding method. In this method, a graphite precursor in flake form is bonded to a glass substrate with the help of an electrostatic field and then cleaved off to leave few layer graphene on the substrate. Now that several methods are available for producing graphene, the relevance of our method is in its simplicity and practicality for producing graphene samples of about $100 \mu \mathrm{m}$ lateral dimensions. This method is also extensible to other layered materials. We discuss some detailed aspects of the fabrication and results from Raman spectroscopy, local probe microscopy, and transport measurements on these samples.
\end{abstract}




\section{Introduction}

The discovery of graphene $[1,2]$ has led to a frenetic development of the field[3], motivated by the spectacular results obtained by various groups and also by the potential that graphene may have with respect to future applications in microelectronics. Several methods [4] have now been developed for producing graphene, including the original methods of epitaxial growth on $\mathrm{SiC}$ substrates[1] and micro-mechanical cleaving[2]. More recent methods include exfoliation [5, 6] and CVD growth on metallic substrates $[7,8]$.

Each of these methods is relevant for specific applications and here we present a method of fabrication of graphene that we have developed which may be classified as a variant of micro-mechanical cleaving. As we have shown in an earlier publication[9] we obtain high quality samples of few layer graphene on Pyrex glass substrates. The quality is seen both from the Raman spectra and from transport measurements. The dimensions that we obtain are typically from a few tens to a few hundred microns for monolayer and bilayer samples while we have obtained trilayer samples of $\mathrm{mm}$ size[9]. A further advantage of this method is that it can be used, as in the case of micromechanical cleaving, for fabricating ultra-thin samples of virtually any layered material in a straightforward manner.

Our aim in this paper is to provide a critical analysis of this method including aspects of fabrication such that the reader may have the necessary elements for replicating it, followed by results of characterization of samples thus obtained. In section 2 we give a brief introduction to the anodic bonding method and its application to the fabrication of ultra-thin samples of layered materials. In section 3 we give results from the Raman characterization of these samples including examples of Raman 'mapping'. In section 4 we show results from local probe microscopy on these samples and discuss some Scanning Tunneling Microscopy (STM) results. Finally in section 5 we discuss some transport measurements on simple Field Effect Transistor (FET) devices.

\section{Anodic bonding and sample fabrication}

Anodic bonding is a much employed technique in the silicon industry [10] and is still widely used [11] for bonding glass to conductive material owing to the good bond quality (silicon-on-insulator bonding or SOI bonding). It allows the joining of two solids without intervening layers like glue and is typically performed between a sodium-bearing glass

substrate (for example Pyrex 7740 from Corning) and a silicon wafer, the two materials being fairly well matched in terms of thermal expansion. At high temperatures, (i $\left.200^{\circ} \mathrm{C}\right) \mathrm{Na}_{2} \mathrm{O}$ in Pyrex glass decomposes into one $\mathrm{O}_{2}^{-}$and two $\mathrm{Na}^{+}$ions. The mobility of the smaller sodium ions is high and the presence of an electric field (the applied potential difference being typically of the order of $1.7 \mathrm{kV}$ ) across the glass substrate [12] makes them migrate towards the cathode at its back. They leave behind a negative space charge in the region of the interface arising from the static oxygen ions. These 
create an electrostatic field with the positive charges in the silicon wafer. Since the space charge layer is typically a few hundred nanometers to a few microns thick, the field created across this layer is very high and pulls the wafer into intimate contact with the substrate, ultimately leading to the formation of stable Si-O-Si bonds and resulting in a wafer bonded to the glass substrate.

Conducting materials which readily oxidize can thus be bonded to Pyrex substrates through a similar mechanism. In the case of graphite the initial bonding is predominantly electrostatic in nature as we will show with the help of our Raman results in section 3 . This is also borne out by the fact that the Raman spectra do not show any sign of formation of graphene oxide. The nature of the two surfaces to be bonded is of importance and a thorough cleaning of the glass substrate with standard laboratory procedure is necessary. We use graphite flake precursors of mineral origin. HOPG graphite for example is not an ideal precursor as graphene obtained from it rarely exceeds lateral dimensions of a few micrometers. We have had good results both from flakes obtained from Madagascar graphite as well as small (typically mm size) graphite inclusions in calcite. The flake must be carefully cleaved before being put into contact with the clean glass surface so as to insure a clean interface. The substrate must contain alkali ions which are mobile under certain conditions and we have used various borosilicate glasses and obtained good results. The surface to be bonded of course needs to be optically polished. Other than these precautions, the bonding can be carried out in normal laboratory surroundings.

We have consistently obtained good results for an interface temperature of 200$250^{\circ} \mathrm{C}$ and an applied potential difference between the cathode on the glass substrate and the anode in contact with graphite in the range of $1700-1800 \mathrm{kV}$. The typical time of application of the potential difference is ten minutes at the end of which the flake is effectively bonded to the substrate. The last step in the procedure is to cleave the flake off the glass substrate using either a scalpel blade or adhesive tape or both. Visual identification with the help of a microscope is then sufficient to isolate graphene remaining on the substrate often in the company of thicker graphite flakes. This identification is somewhat more difficult than in the case of a $\mathrm{SiO}_{2} / \mathrm{Si}$ substrate because it is based only on optical contrast. This is the reason that our optical micrographs do not resembles the 'standard' ones for graphene on $\mathrm{SiO}_{2} / \mathrm{Si}$ substrates. With experience one can identify monolayer samples, and few layer samples are easily visible.

\section{Raman spectroscopy}

Raman spectroscopy has fast become a method of choice both for characterizing graphene samples as shown by several early papers $[13,14]$, as well as studying electron phonon coupling in graphene [15] and its variation with doping. Indeed the $2 \mathrm{D}$ lineshape is very sensitive to the number of layers in the graphene sample. The lineshape also depends on the nature of the substrate [16,9], typically in a bilayer the multiple peak nature is more obvious in graphene bilayers on $\mathrm{SiO}_{2} / \mathrm{Si}$ substrates. Here we show 


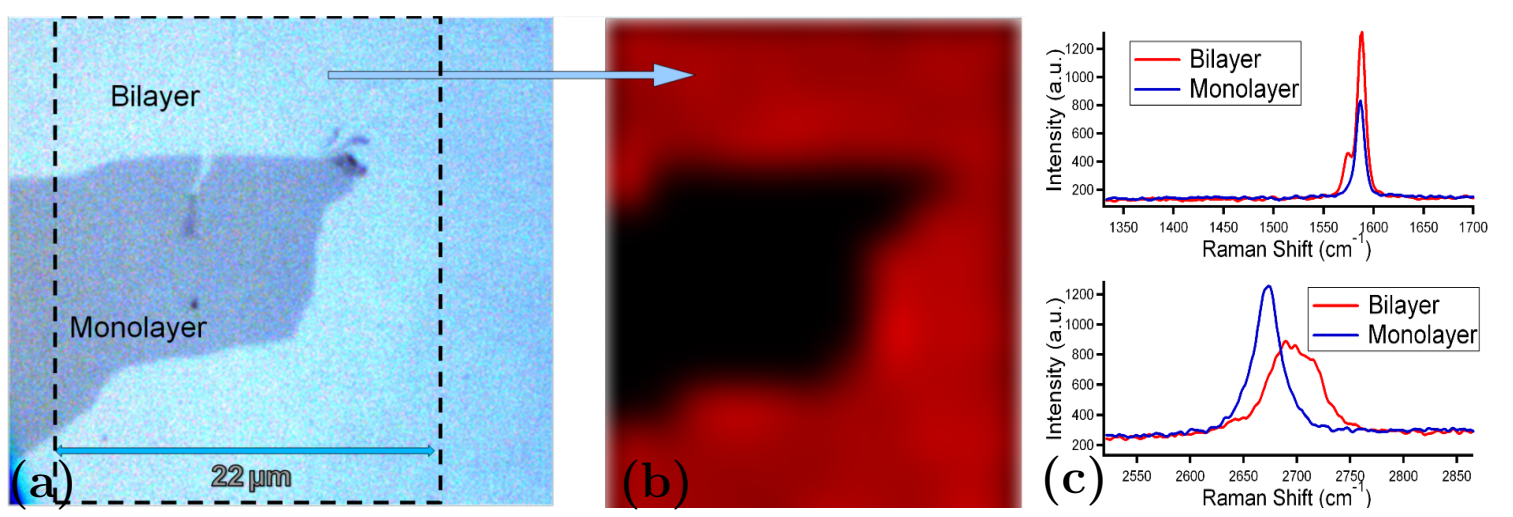

Figure 1. (a) Optical micrograph of a graphene monolayer surrounded by a bilayer. (b) Raman map of a part of the sample shown in a) The width of the 2D line was used to define the colour code giving black for the monlayer area and red for the bilayer area. (c) representative Raman spectra for the $\mathrm{G}$ line and the $2 \mathrm{D}$ line from the monolayer and the bilayer.

two examples of the application of Raman mapping of our graphene samples. Raman maps and spectra were acquired using a Renishaw InVia Reflex microspectrometer. A $514 \mathrm{~nm}$ Laser Physics argon laser was focused on the sample by a DMLM Leica microscope with a 50x $(\mathrm{NA}=0.75)$ objective. The Rayleigh diffusion was eliminated by edge filters and the signal was finally dispersed using a $1800 \mathrm{gr} / \mathrm{mm}$ grating. In the first example we show how optical identification of graphene is corroborated by Raman measurements. In the second we show measurements on a sample which is doped insitu due to strong electrostatic interaction with the substrate. It is now well-known that doping of graphene provokes a shift in the $\mathrm{G}$ and 2D lines $[17,18]$. The $\mathrm{G}$ line shift is a reliable method for extracting a corresponding doping level. Notably, in bilayer graphene doping can induce a splitting of the $\mathrm{G}$ line [18].

In Figure 1(a) we show an optical micrograph of a graphene sample which is a monolayer island (darker part of the image) inside a bilayer. The Raman map of a part of the sample is shown in Figure 1(b). The color code corresponds to the width of the 2D line and clearly demarcates the bilayer part from the monolayer. The representative linehapes for this sample are shown in Figure 1(c), both for the $\mathrm{G}$ line and the 2D line.

In Figure 2(a) we show an optical monograph of a large monolayer sample with small parts of bilayer and thicker graphene towards the top edge. In Figure 2(b) we show the corresponding Raman map of the sample where the colour code is based on fits of the measured spectra in the terms of reference spectra for monolayer (dark blue), bilayer (light blue) and few layer (white). Here we find that both the $\mathrm{G}$ and 2D lines are consistently shifted with respect to the values expected for undoped graphene.

In Figure 2(c) we show representative spectra for the $\mathrm{G}$ line and the 2D line in the monolayer, bilayer and few layer parts of the sample. The few layer graphene has an average thickness of $2 \mathrm{~nm}$ as measured by Atomic Force Microscopy (AFM) and should correspond to four layers. The shift in the Raman lines is considerable (G peak at 1611 $\mathrm{cm}^{-1}, 2 \mathrm{D}$ peak at $2706 \mathrm{~cm}^{-1}$ as seen, for example in comparison to the values shown for 

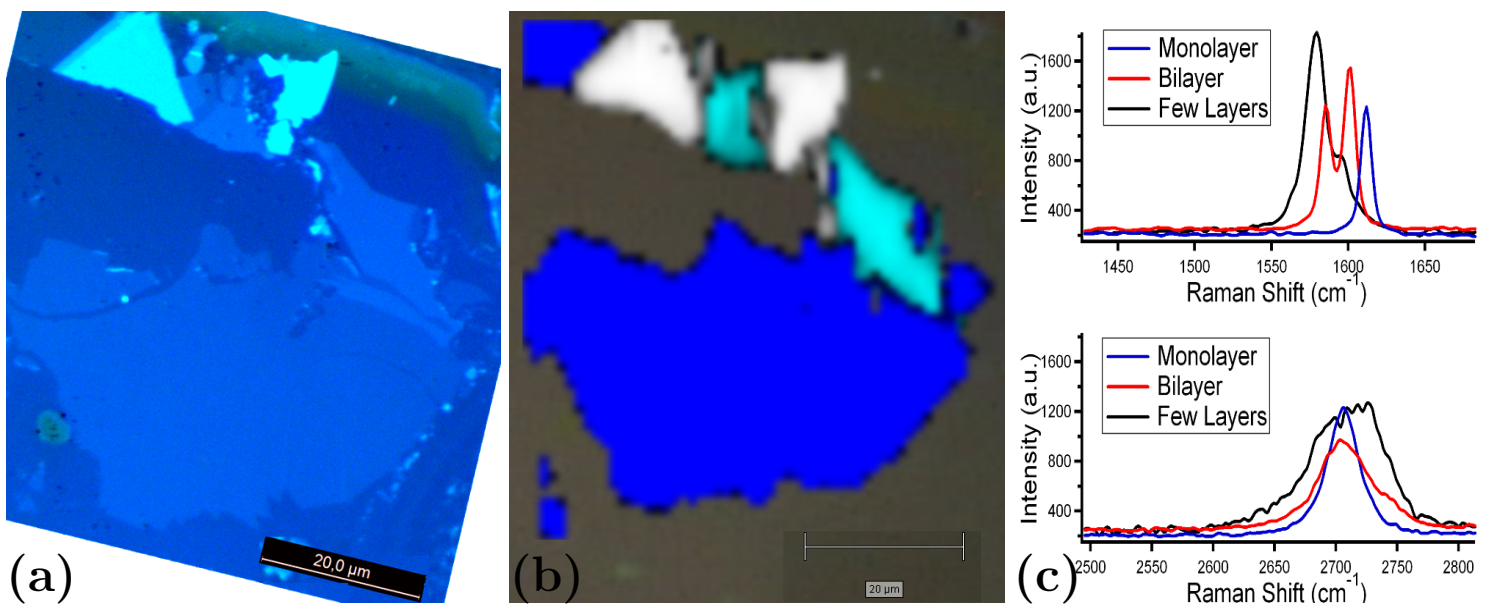

Figure 2. (a) Optical micrograph of a monlayer sample with thicker graphene layers towards the top edge of the photo. (b) Raman map of the same sample where the colour code is determined by fitting the spectrum measured at each point with respect to spectra measured on the monolayer (dark blue), bilayer (light blue) and few layer (white) parts of the sample. (c) Representative Raman spectra from the three area in the sample.

the first sample in Figure 1 and by comparison with theoretical estimations and earlier experimental data of doping as a function of gate voltage $[18,19,17]$ we estimate the number of doped holes to be $4.5 \times 10^{13} \mathrm{~cm}^{-2}$. Most interestingly, we find that the bilayer $\mathrm{G}$ line is consistently split $\left(1585 \mathrm{~cm}^{-1}\right.$ and $\left.1600 \mathrm{~cm}^{-1}\right)$ as predicted by calculations and the intrinsic doping estimation from the shifts of the $\mathrm{G}$ and 2D lines for the monolayer as well as the bilayer is coherent.

Graphene samples are often intrinsically doped due to interaction with the susbstrate[16]. In our samples in general we do not find large shifts of the Raman lines. However, since our samples are bonded due to an electrostatic interaction we can expect enhanced interaction with the charged substrate, though one would expect this charging and hence the interaction to decay over time. We have found large shifts in the Raman lines in a few samples, as the one presented here. The enhanced intrinsic doping is a strong indication of the electrostatic nature of the bonding process in our samples as the space charge layer formed at the interface during the bonding acts as a polarized bottom gate.

\section{Local probe microscopy}

We performed room temperature UHV STM imaging on some of our samples and observed different phenomena related to defects and layer decoupling. The samples were initially earthed with photolithographically deposited gold contacts. However photolithography was found to considerably degrade the sample surface requiring additional annealing and cleaning and we now prefer to use mechanical masks to evaporate the gold contacts onto the sample and substrate. 
In Figure 3(a) we show a large sample of graphene (about $100 \mu \mathrm{m}$ in lateral dimensions). The darkest portions of the image correspond to the glass substrate and the lighter parts correspond to graphene layers. Raman spectra measured on two spots on this thinnest layer are shown in Figure 3(c). The measured lineshape of the 2D line resembles that of a single peak and hence one could conclude that the sample is a monolayer. However the full width at half maximum (FWHM) is large $\left(48 \mathrm{~cm}^{-1}\right)$ and could indicate a bilayer since the bilayer $2 \mathrm{D}$ line is a composite of more than one peak. The issue is resolved by confrontation with the surface topography obtained by AFM and with the images of the local density of states obtained by STM. An AFM scan of a portion of the sample indicated by a square in Figure 3(a), is shown in Figure 3(b). The average step height measured between the graphene and the substrate is about 1.2 $\mathrm{nm}$ indicating that we have indeed a bilayer sample.
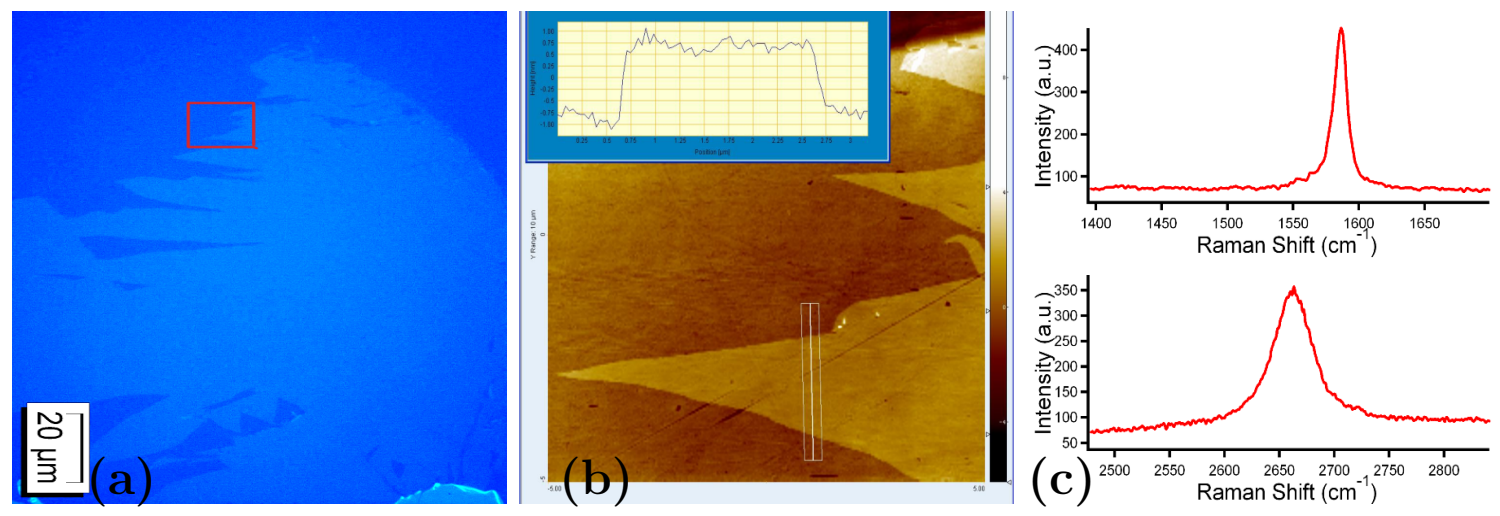

Figure 3. (a) Optical micrograph of a graphene sample used for STM imaging. (b) AFM imaging of the part of the sample shown in the red rectangle in (a). The step size with respect to the substrate indicates a height $(1.2 \mathrm{~nm}$, see profile in the inset) corresponding to a bilayer. (c) Respresentative Raman spectra from the sample. The 2D lineshape is that of a single Lorentzian and would indicate a monlayer, but the width suggests a thicker sample. The AFM measurement in (b) clearly points to a bilayer sample and the lineshape is explained by partial decoupling between the layers due to rotational misalignment (see text).

In Figure 4 we present some room temperature UHV STM images of our graphene samples. Figure 4(a) represents a moiré pattern obtained on the sample in Figure 3, confirming that it cannot be single layer graphene since the moiré pattern originates from a rotational misalignment between two superimposed graphene layers. In the large area STM scan shown here (the spots are not those of the equivalent graphite lattice, the lateral dimension of the scan being $90 \mathrm{~nm}$ ) we observe two adjacent moiré domains differing by the periodicity of the spots and thus by the interlayer rotational misalignment. The relation[20] between the period D of the supperlattice and the rotation angle $\theta$ of the two layers is $\mathrm{D}=\frac{d}{2 \sin \theta / 2}$, where $\mathrm{d}$ is the period of the original lattice. The upper domain has a lattice parameter of $\mathrm{a}=11 \mathrm{~nm}$ and a misalignment angle $\theta=2.56^{\circ}$, and the lower domain a lattice parameter of $15 \mathrm{~nm}$ and a misalignment angle $\theta=1.87^{\circ}$. This also explains why the Raman 2D line has a lineshape reminiscent 
of the monolayer since this interlayer rotation would also electronically decouple one layer from the other. Other moiré patterns were also observed, with lattices parameters from $2.2 \mathrm{~nm}$ up to $42 \mathrm{~nm}$, and corresponding to rotational angles from $12.84^{\circ}$ to $0.67^{\circ}$.
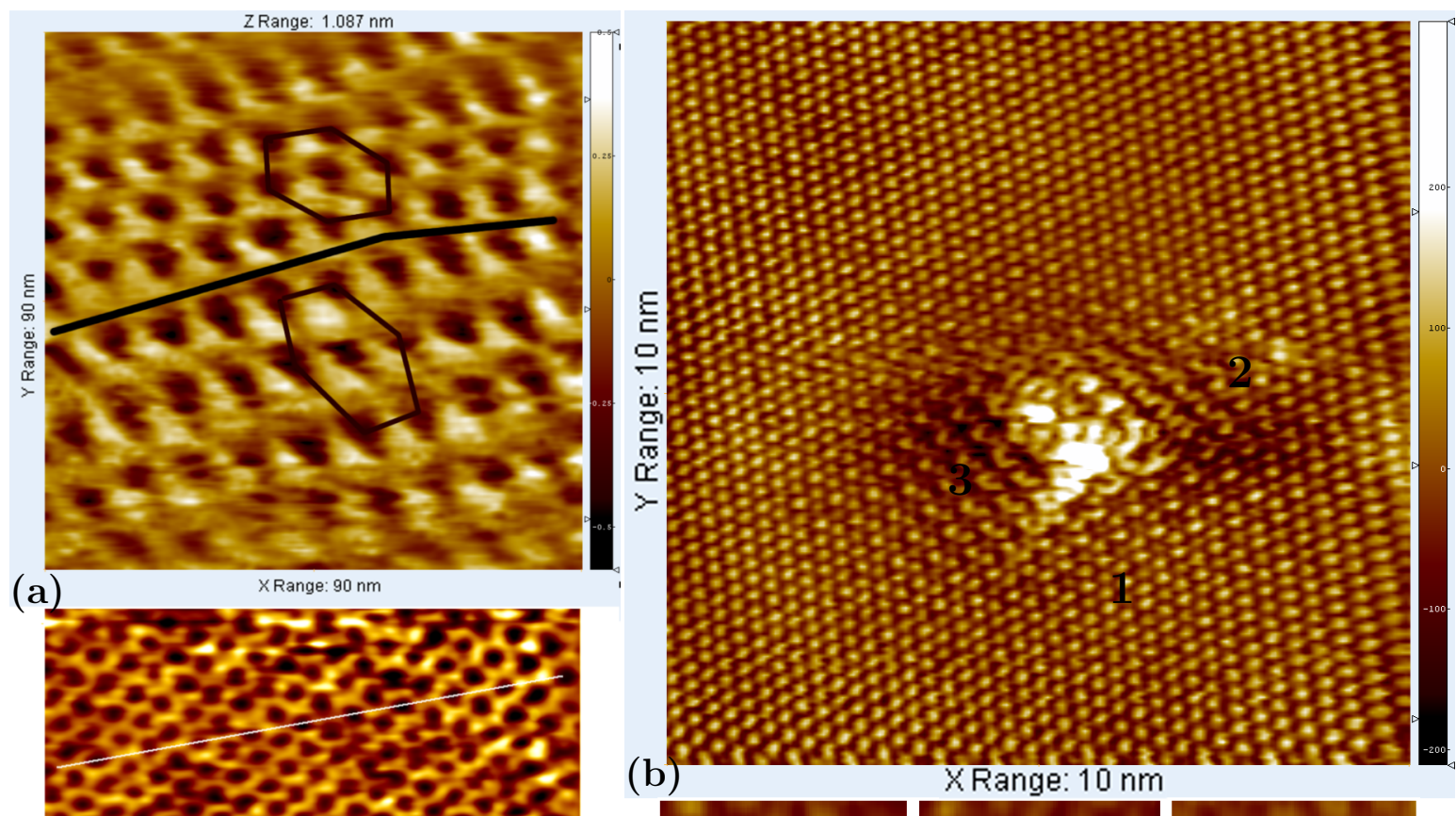

(a)
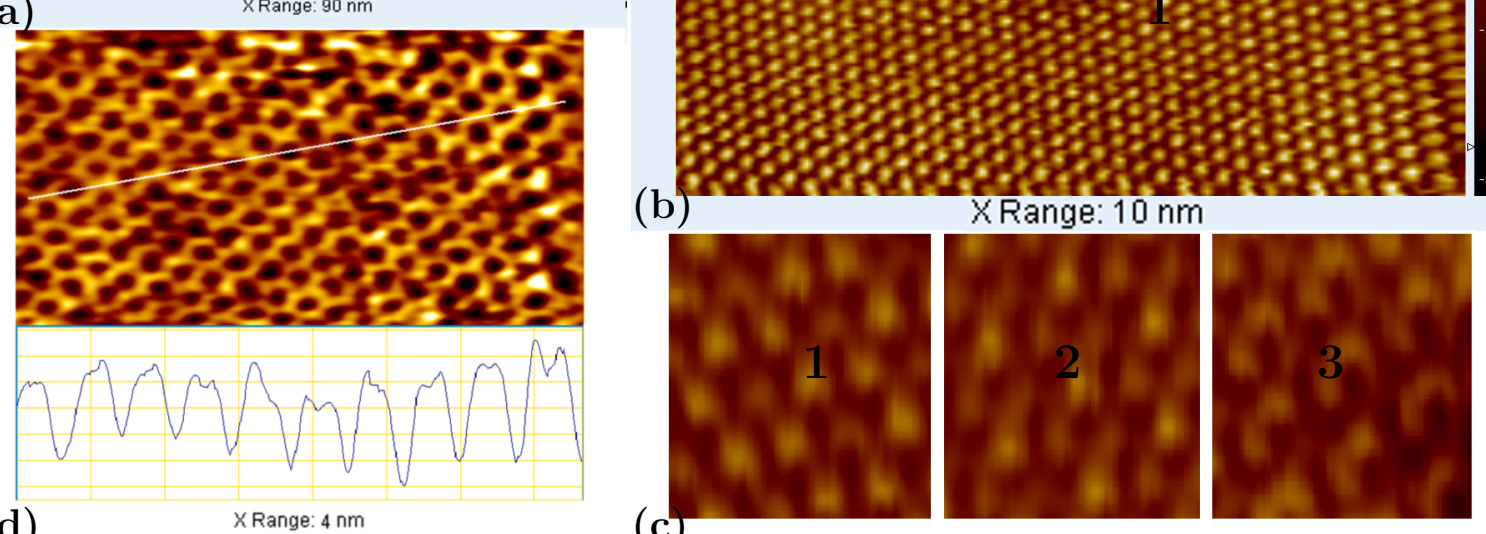

Figure 4. (a) moiré domains imaged on the bilayer, indicating rotational misalignment between the layers for which we can calculate the angle (see text). (b) Fe atoms were deposited on the surface of the samples and on a thicker edge corresponding to few layer graphene we could observe perturbations in the local density of states introduced by an isolated defect. These perturbations are seen to be screened over a distance of 2-4 $\mathrm{nm}$ yielding the equivalent graphite lattice away form the defect. (c) A zoom into three areas from the image in (b) showing different surface patterns for the local density of states. Area 1 corresponds to the $\sqrt{3} \mathrm{x} \sqrt{3} \mathrm{R} 30$ LDOS surface pattern while areas 2 and 3 are more subtle variations of this electronic reconstruction. (d) A monolayer graphene sample should have a signature honeycomb STM image and this is what we observe in this figure. The inset show a profile along the white line in the figure showing neighboring carbon atoms.

In order to investigate the effect of local perturbations on the measured Local Density of States (LDOS) in graphene we deposited a small quantity of Fe (nominally $0.2 \mathrm{ML}$ ) on this graphene sample. On large scale STM images the appearance of scattered dots confirmed the presence of Fe atoms or clusters on the graphene surface, similar to those observed on iron doped graphite[21]. On a thicker edge of the sample corresponding to few layer graphene, we were able to image periodic oscillations in 
the local density of states induced by an isolated defect corresponding very probably to a Fe cluster (Figure 4(b)). The defect induces oscillations with a global three fold symmetry corresponding to similar earlier observations [22]. The supperlattices formed have a lattice parameter $\sqrt{3}$ times greater and are rotated with $30^{\circ}$ with respect to the graphite lattice $(\sqrt{3} \mathrm{x} \sqrt{3} \mathrm{R} 30$ LDOS reconstruction). These oscillations are screened out away from the defect over distances of 2-4 $\mathrm{nm}$.

The rich screening pattern surrounding such defects, has attracted theoretical attention in the past in the case of graphite and requires the calculation of local density of states. This is obtained by solving for the electronic structure of the graphite/graphene lattice with the relevant local perturbations (a non trivial problem). We observe here that the internal structure of the defect (a closer look will reveal that there are two maxima in the STM image of the defect) provokes different patterns in the oscillations as explained by Mizes et al. [22]. In Figure 4(c) we show three different examples of these patterns.

Finally in Figure 4(d) we show an STM image of the expected honeycomb structure from a monolayer graphene sample. Though this honeycomb structure is a signature of an uncoupled graphene layer we have also observed it in few layer graphene, and even in graphite. This is possible [23] if the imaged portion under the STM tip is indeed decoupled from the underlying layers.

\section{Transport measurements}

In this last section we give typical results for transport measurements conducted on simple field effect transistor devices made from anodic bonded graphene. We show device characteristics of a top-gated monolayer (inset of Figure 5(b)) and bilayer (inset of Figure 5(a)) graphene samples. These were patterned using photolithography and $\mathrm{Au} / \mathrm{Cr}(30 / 10 \mathrm{~nm})$ bilayer deposition. By measuring the resistance in two and fourpoint configurations the average contact resistance is estimated to be of the order of 50 ohms. For the gating of our devices we use electrochemical top-gating with a solid (Polyethylene oxide/ $\mathrm{LiClO}_{4}$ ) electrolyte [17] which allows high doping levels with low gate voltages. On the addition of the electrolyte the sample resistance increases, possibly due to the addition of impurities.

In Figure 5 we show the square resistance of our devices calculated using the corresponding aspect ratio. The charge neutrality peak, characteristic of few-layer graphene, is found at gate voltages of $0.51 \mathrm{~V}$ for the monolayer and $0.3 \mathrm{~V}$ for the bilayer. I-V characteristics (shown for the bilayer) between source and drain show linear behavior, excluding Schottky barriers at the contacts, and expectedly reflect the drop in resistance due to the field effect doping.

The solid electrolyte is based on the diffusion of Li ions and on the application of a reasonable (generally 2 volts or below) voltage between the gate and the source or drain electrode. The charge appearing on the gate electrode causes ionic diffusion in the solid electrolyte and the formation of a Debye layer over a characteristic distance 
corresponding to charge separation for the electrolyte. This distance is typically 1 or $2 \mathrm{~nm}$ for our case and since the Debye layer screens the applied field, it is also the effective distance over which the field is applied to the graphene sample. It can be seen that this field can be almost one order of magnitude higher with respect to standard dielectrics used since the latter are often much thicker. This is a considerable advantage. However there are also some drawbacks including the fact that the diffusion of Li ions is suppressed at low temperatures which means that to change the doping in the sample, one has to apply the corresponding gate voltage at room temperature.
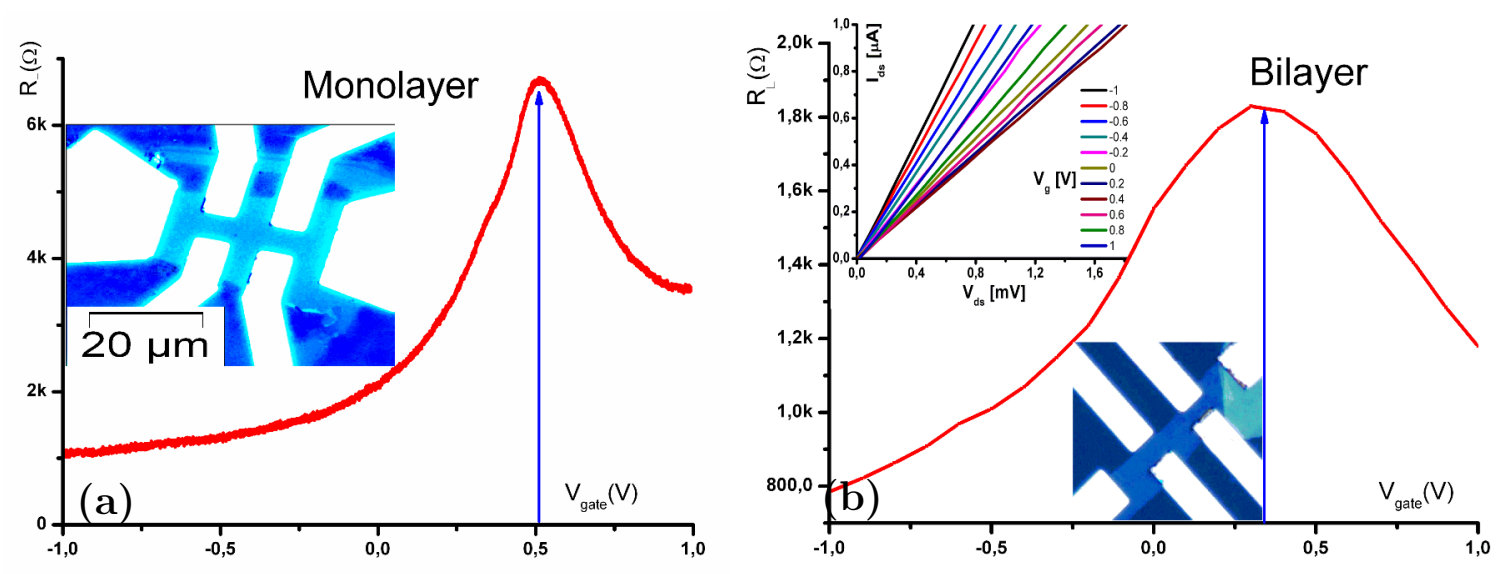

Figure 5. Resistance as a function of top gate voltage for devices pictured in the inset. In the inset for figure 5 (b)we show as measured I-V characteristics as a function of the gate voltage. (a) bilayer, (b) monolayer.

\section{Conclusion and Perspectives}

The straightforward question that one can ask is the relevance of another method for making graphene. We hope to have shown that the method that we present is a simple and practical way of producing good quality and large area graphene on an insulating substrate for laboratory purposes. The graphene can only be as 'good' as the precursor used and the maximum size that we can hope to obtain for our samples is also limited by the quality of the precursor which means roughly mm size. This is however largely sufficient for most laboratory and research applications. We are now working on ways of transposing these graphene samples on other substrates, with a preference for mechanical transposition since chemical treatment often leaves unwanted residue on the sample which alters its electronic and mechanical properties.

Finally, as we have already stated, the method can also be used for producing ultra-thin films of other layered substances. We have prepared few atomic layer thick films of $\mathrm{NbSe}_{2}$ and InSe for example and this aspect is particularly interesting. In only a few years graphene has become a huge and well trodden research field. However the 
discovery of graphene has opened up the general field of two dimensional materials and their intriguing properties and this field is still largely unexplored.

We acknowledge assistance from F. Gelebart and M. Morand, discussions with V. Etgens and F. Mauri and funding from DRITT(UPMC) and by E.U. EuroMagNet II program under contract 228043.

\section{References}

[1] Claire Berger, Zhimin Song, Tianbo Li, Xuebin Li, Asmerom Y. Ogbazghi, Rui Feng, Zhenting Dai, Alexei N. Marchenkov, Edward H. Conrad, Phillip N. First, and Walt A. de Heer. Ultrathin epitaxial graphite: 2d electron gas properties and a route toward graphene-based nanoelectronics. The Journal of Physical Chemistry B, 108(52):19912-19916, Dec 2004. doi: 10.1021/jp040650f.

[2] K. S. Novoselov, D. Jiang, F. Schedin, T. J. Booth, V. V. Khotkevich, S. V. Morozov, and A. K. Geim. Two-dimensional atomic crystals. Proceedings of the National Academy of Sciences of the United States of America, 102(30):10451-10453, 2005.

[3] A. H. Castro Neto, F. Guinea, N. M. R. Peres, K. S. Novoselov, and A. K. Geim. The electronic properties of graphene. Rev. Mod. Phys., 81(1):109-162, Jan 2009.

[4] A. K. Geim. Graphene: Status and Prospects. Science, 324(5934):1530-1534, 2009.

[5] Yenny Hernandez, Valeria Nicolosi, Mustafa Lotya, Fiona M. Blighe, Zhenyu Sun, Sukanta De, McGovernI T., Brendan Holland, Michele Byrne, Yurii K. Gun'Ko, John J. Boland, Peter Niraj, Georg Duesberg, Satheesh Krishnamurthy, Robbie Goodhue, John Hutchison, Vittorio Scardaci, Andrea C. Ferrari, and Jonathan N. Coleman. High-yield production of graphene by liquid-phase exfoliation of graphite. Nat Nano, 3(9):563-568, Sep 2008.

[6] Dmitriy A. Dikin, Sasha Stankovich, Eric J. Zimney, Richard D. Piner, Geoffrey H. B. Dommett, Guennadi Evmenenko, SonBinh T. Nguyen, and Rodney S. Ruoff. Preparation and characterization of graphene oxide paper. Nature, 448(7152):457-460, Jul 2007.

[7] Alfonso Reina, Xiaoting Jia, John Ho, Daniel Nezich, Hyungbin Son, Vladimir Bulovic, Mildred S. Dresselhaus, and Jing Kong. Large area, few-layer graphene films on arbitrary substrates by chemical vapor deposition. Nano Letters, 9(1):30-35, Dec 2008. doi: 10.1021/nl801827v.

[8] Keun Soo Kim, Yue Zhao, Houk Jang, Sang Yoon Lee, Jong Min Kim, Kwang S. Kim, JongHyun Ahn, Philip Kim, Jae-Young Choi, and Byung Hee Hong. Large-scale pattern growth of graphene films for stretchable transparent electrodes. Nature, 457(7230):706-710, Feb 2009.

[9] Abhay Shukla, Rakesh Kumar, Javed Mazher, and Adrian Balan. Graphene made easy: High quality, large-area samples. Solid State Communications, 149(17-18):718-721, 2009.

[10] George Wallis and Daniel I. Pomerantz. Field assisted glass-metal sealing. Journal of Applied Physics, 40(10):3946-3949, 1969.

[11] Emilie Collart and Abhay Shukla Marc Morand Ccile Malgrange Nathalie Bardou Ali Madouri Jean-Luc Pelouard Frdric Glbart. Spherically bent analyzers for resonant inelastic x-ray scattering with intrinsic resolution below 200 mev. Journal of Synchrotron Radiation, 12(4):473$478,2005$.

[12] Kevin B. Albaugh. Electrode phenomena during anodic bonding of silicon to sodium borosilicate glass. Journal of The Electrochemical Society, 138(10):3089-3094, 1991.

[13] A. C. Ferrari, J. C. Meyer, V. Scardaci, C. Casiraghi, M. Lazzeri, F. Mauri, S. Piscanec, D. Jiang, K. S. Novoselov, S. Roth, and A. K. Geim. Raman spectrum of graphene and graphene layers. Phys. Rev. Lett., 97(18):187401, Oct 2006.

[14] D. Graf, F. Molitor, K. Ensslin, C. Stampfer, A. Jungen, C. Hierold, and L. Wirtz. Spatially resolved raman spectroscopy of single- and few-layer graphene. Nano Letters, 7(2):238-242, Jan 2007. doi: $10.1021 /$ nl061702a. 
[15] Nicola Bonini, Michele Lazzeri, Nicola Marzari, and Francesco Mauri. Phonon anharmonicities in graphite and graphene. Phys. Rev. Lett., 99(17):176802, Oct 2007.

[16] C. Casiraghi, S. Pisana, K. S. Novoselov, A. K. Geim, and A. C. Ferrari. Raman fingerprint of charged impurities in graphene. Applied Physics Letters, 91(23):233108, 2007.

[17] Das A., Pisana S., Chakraborty B., Piscanec S., Saha S K., WaghmareU V., NovoselovK S., KrishnamurthyH R., GeimA K., FerrariA C., and SoodA K. Monitoring dopants by raman scattering in an electrochemically top-gated graphene transistor. Nat Nano, 3(4):210-215, Apr 2008.

[18] Paola Gava, Michele Lazzeri, A. Marco Saitta, and Francesco Mauri. Probing the electrostatic environment of bilayer graphene using raman spectra. Phys. Rev. B, 80(15):155422, Oct 2009.

[19] Paola Gava, Michele Lazzeri, A. Marco Saitta, and Francesco Mauri. Ab initio study of gap opening and screening effects in gated bilayer graphene. Phys. Rev. B, 79(16):165431, Apr 2009.

[20] J. M. Campanera, G. Savini, I. Suarez-Martinez, and M. I. Heggie. Density functional calculations on the intricacies of moiré patterns on graphite. Phys. Rev. B, 75(23):235449, Jun 2007.

[21] E. Bourelle, Y. Kaburagi, Y. Hishiyama, and M. Inagaki. Stm study of surfaces of kish graphite doped by iron. Carbon, 39(13):1955 - 1962, 2001.

[22] H. A. MIZES and J. S. FOSTER. Long-Range Electronic Perturbations Caused by Defects Using Scanning Tunneling Microscopy. Science, 244(4904):559-562, 1989.

[23] Guohong Li, Adina Luican, and Eva Y. Andrei. Scanning tunneling spectroscopy of graphene on graphite. Phys. Rev. Lett., 102(17):176804, Apr 2009. 